Accretion Phenomena and Related Outfows, IAU Colloquium 168

ASP Conference Series, Vol. 121, 1997

D.T. Wickramasinghe, L. Ferrario, and G.V. Bicknell, eds.

\title{
X-ray Observations of Accretion Disks
}

\author{
Nicholas E. White \\ Code 662, Laboratory for High Energy Astrophysics, \\ NASA Goddard Space Flight Center, \\ Greenbelt, MD, 20769 USA.
}

\begin{abstract}
.
The properties of accretion disks in low mass X-ray binaries (LMXRB) are reviewed in the context of recent results from the $A S C A$ observatory.
\end{abstract}

\section{Introduction}

The low mass X-ray binary (LMXRB) systems are neutron stars or black holes that accrete material from a late type companion star that fills its critical potential Roche lobe. The observed properties of the high inclination LMXRB are dominated by the effects of the X-ray heated accretion disk and the disk/gas stream interaction. Observations of the X-ray emission from the central compact $\mathrm{X}$-ray source in high inclination systems has given a detailed observational picture of the accretion disk structure (see e.g. White, Nagase and Parmar 1995). There appears to a very turbulent region at the edge of the disk caused by the impact of the gas stream from the companion, where material is projected well above the disk plane. Another important effect that has been identified is that X-ray illumination of the accretion disk results in the formation of an accretion disk corona (ADC), which may be optically thick when viewed at high inclinations. This review will describe the current observations and theoretical understanding of accretion disks in LMXRB. It begins with an overview of the disk/Gas Stream interaction and then describes recent $A S C A$ spectral results.

\section{The Disk/Gas Stream Interaction in LMXRB}

LMXRB show periodic dipping behaviour that is from absorption by material projected up above the disk plane where the gas stream from the companion hits the accretion disk. The highest quality observations of these systems come from the EXOSAT observatory whose long $90 \mathrm{hr}$ orbit allowed uninterupted observations of these systems. Figure 1 shows three examples of dipping LMXRB sources. The lightcurves show similar intervals of dipping that lasts $\sim 10-20 \%$ of an orbital cycle. Where an optical counterpart is seen there is a strong orbital modulation caused by both X-ray heating of the companion and/or an eclipse of the $\mathrm{X}$-ray heated accretion disk. The relative phase of the dipping activity is consistent with the expected location of the gas/stream interaction. In some cases (not shown) a second less intense interval of dipping is seen at an orbital phase opposite to the main interval of dipping that may be caused 


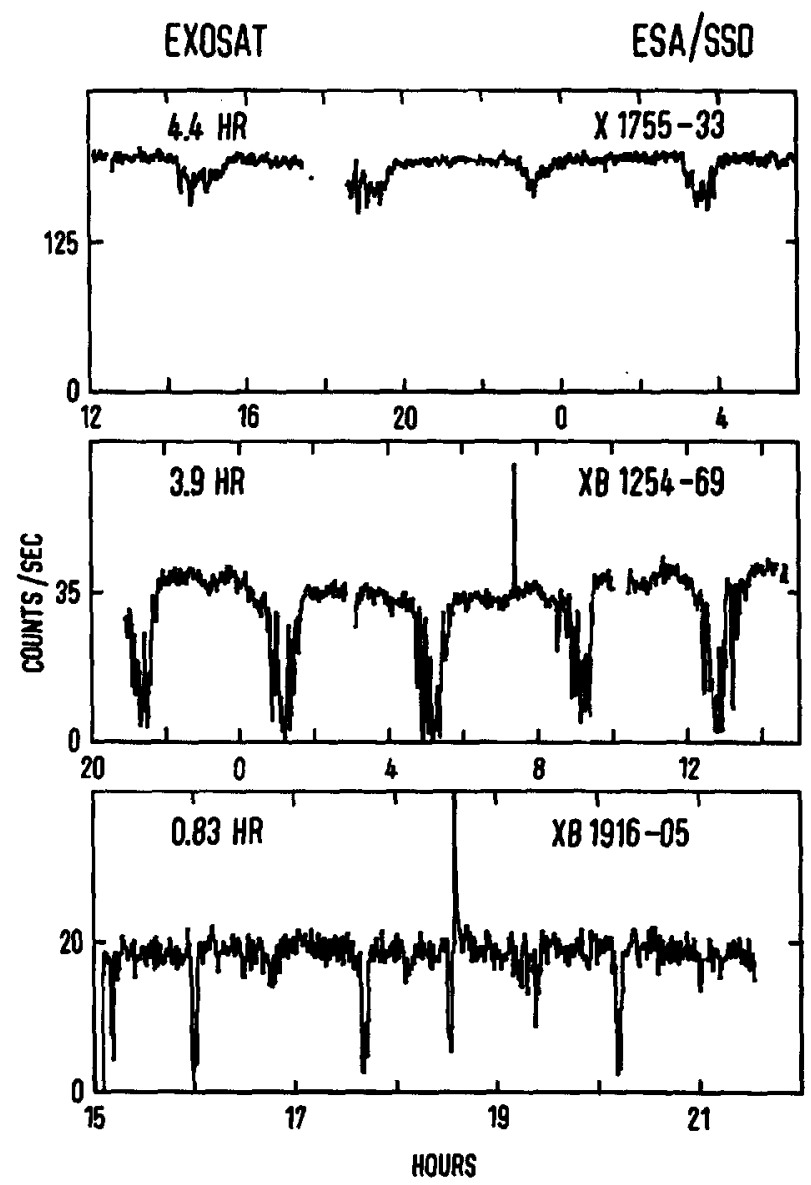

Figure 1. The EXOSAT lightcurves of three moderate to high inclination LMXRB sources. These three sources show periodic dipping activity that is caused by the disk/gas stream interaction region passing through the line of sight. The systems do not show eclipses from the companion star, which indicates the inclinations are greater than $\sim 80^{\circ}$. The spikes are type I X-ray bursts. The difference in the depth of the dips may reflect abundance differences between the systems, or the residual emission from an extended ADC region (see White, Nagase and Parmar 1995 and refs therein). 
by a second interaction with the gas stream on the opposite side of the disk. Two dipping sources also show a very brief (few minutes) eclipse by the companion star: EXO 0748-676 (Parmar et al. 1986) and 4U1658-29 (Cominsky and Wood 1984). The orbital lightcurve of EXO 0748-676 is shown in Figure 2. The eclipse follows an interval of dipping activity, again confirming that the dips are due to the splash from the accretion stream passing through the line of sight.

Migrom (1978) suggested that the fewer than expected X-ray eclipses from LMXRB could be explained if LMXRB contain thick accretion disks which block the X-ray source in systems that are viewed at high inclinations. The discovery of a partial eclipse with HEAO 1 from 4U1822-37 proved Milgrom's thick-disk model to be correct (White et al. 1981). The orbital lightcurve is shown in Figure 2. The $4 \mathrm{U} 1822-37$ system is viewed almost edge on, with the compact X-ray source probably hidden behind the disk rim. X-rays are scattered in a photoionized accretion disk corona (ADC). The $\mathrm{ADC}$ has a much larger extent than that of the central compact object and this results in a partial X-ray eclipse and a relatively low X-ray luminosity $\sim 100$ times less than typically seen for LMXRB. The orbital light curve of $4 \mathrm{U} 1822-37$ also shows a sinusoidal modulation (Figure 2 ), with a minimum preceding the partial eclipse that is ascribed to the partial occultation of the accretion disk corona, $\mathrm{ADC}$, by a bulge at the rim of the disk caused by its interaction with the incoming gas stream (White and Holt 1982). Multiwavelength modelling of the orbital lightcurves in many bands has allowed a very detailed picture of the 4 U1822-37 system to be built up (White and Holt 1982, Mason and Córdova 1982, Hellier and Mason 1989). This shows that the $\mathrm{X}$-ray scattering region is about one third of a solar radius, and is about half the size of the accretion disk. There is also a considerable thickening of the disk at the rim with a maximum thickness at the impact point of the gas stream, with a secondary maximum on the opposite side of the disk.

While it is has been accepted that the dipping behaviour from the LMXRB is due to absorption from material stirred up from the disk plane by the interaction of the gas stream with the disk, the details of this process are complex and theoretical modelling of the lightcurves has lagged far behind the observations. Pioneering calculations made by Lubow and Shu (1975) showed that a significant fraction of the gas stream may flow over the accretion disk surface in a ballistic trajectory before circularizing at a radius determined by the angular momentum of the flow. This is significantly smaller than the size of the disk, and could result in a ring like thickening at this radius. Following this Frank, King, and Lasota (1987) suggest that material in the gas stream strikes this ring, causing a bulge that extends about half way around the disk between orbital phase 0.3 and 0.8 . More recently Armitage and Livio (1996) have used three dimensional Smooth Particle Hydrodynamic simulations to investigate in detail the interaction of the gas stream with the disk and to make detailed predictions that can be compared with the observations. They find that a significant fraction of the stream material can ricochet off the disk edge and overflow to smaller radii. The predictions from the modelling of Armitage and Livio (1996) of column density vs. orbital phase for different inclinations appears to be in good qualitative agreement with the observed lightcurves, including the secondary dips seen out of phase with the main activity. 


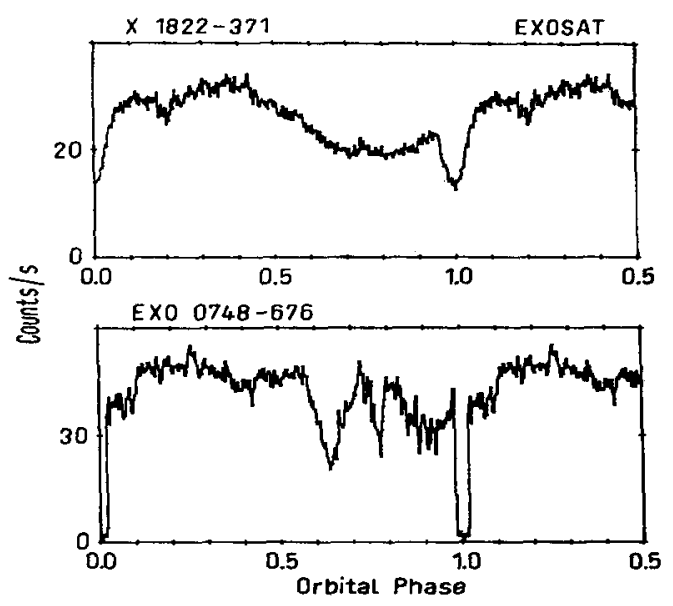

Figure 2. The orbital lightcurves of two eclipsing LMXRB systems. For the $5.57 \mathrm{hr}$ period system $4 \mathrm{U} 1822-37$ the compact X-ray source is blocked by accretion disk rim resulting in a partial eclipse. Material associated with the disk/gas stream interaction causes a secondary minimum that leads the eclipse. In the case of the $3.8 \mathrm{hr}$ LMXRB EXO 0748-676 the system parameters are such that both dipping and a brief total eclipse are seen.

\section{Accretion Disk Coronae in LMXRB (ADC)}

An ADC is the result of the illumination of the surrounding accretion disk by the central compact X-ray source. Hot material is evaporated from the disk surface and gravitationally bound to the disk by the mass of the central neutron star or black hole. The existence of ADC in X-ray binaries had first been predicted by Shakura and Sunyaev (1972). The first direct evidence for their existence came from the discovery of an extended eclipse in the binary 4U1822-37 by White et al. (1981). The vertical structure of an ADC is analogous to that of the solar corona, with a cool photosphere, a chromosphere and corona. The major difference between the solar corona and an ADC is in the latter the energy source comes from above and magnetic fields are not required to confine the hot material. Like the solar corona, an $\mathrm{ADC}$ is expected to be a rich source of $\mathrm{X}$-ray line emission. In an ADC the lines comes from fluorescence of the photosphere by the central X-ray source and recombination in the chromosphere/coronal regions (e.g. Ko and Kallman 1994). The spectra of many LMXRB show low energy line emission between 0.6 and $1.1 \mathrm{keV}$ from the iron $\mathrm{L}$ shell and the $\mathrm{K}$ shell of oxygen, nitrogen and other medium-Z elements (e.g. Kahn, Seward and Chlebowski 1984; Vrtilek et al. 1986, 1991; Christian, White and Swank 1993). Broad iron $\mathrm{K}$ line at $6.7 \mathrm{keV}$ is also seen (Suzuki et al. 1984; White et al. 1986) that may come from recombination in an ADC (Hirano et al. 1987; Kallman and White 1989). 


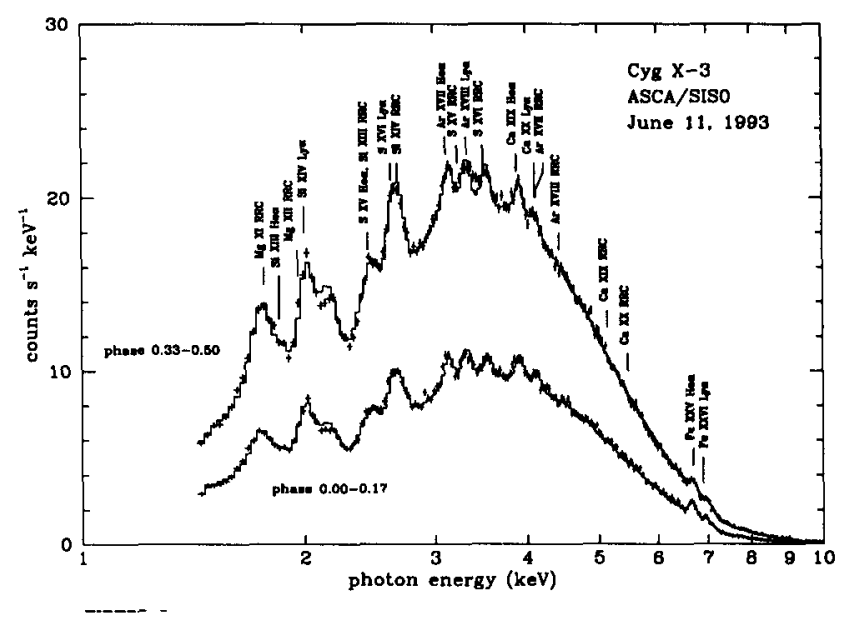

Figure 3. The ASCA SIS spectra of Cyg X-3 from Liedahl and Paerels (1996). The upper and lower traces come from the low and high portions of the $4.8 \mathrm{hr}$ orbital cycle. The various line features include the first clear detection of radiative recombination edges in the spectrum of a cosmic X-ray source.

The emission features, lines and recombination edges in the $0.8-10 \mathrm{keV}$ region of the spectrum are dominated by the transitions from from the $\mathrm{L}$ shell of various ions of iron and from the $K$ shells of the most abundant elements such as $\mathrm{N}, \mathrm{O}, \mathrm{Ne}, \mathrm{Mg}, \mathrm{S}, \mathrm{Si}, \mathrm{Ca}, \mathrm{Ar}$ and $\mathrm{Fe}$. In a photo-ionized gas radiative recombination (RRC) is the dominant mechanism for populating excited levels (e.g. Liedahl et al. 1992). The relative efficiency of resonant excitation to radiative recombination can dramatically effect the observed line emission. Resonant scattering is an elastic Process and a stationary spherical scattering cloud with a point source embedded in it will produce no net line photons. However, if the line of sight to the continuum source is blocked, or the cloud is asymmetric (e.g. in an ADC) then only the emission part of the line will appear. A similar effect will occur in a wind because the scattered emission and absorption will appear at different observed wavelengths.

An observation of Cyg X-3 by $A S C A$ revealed for the first time prominent lines from $\mathrm{H}$ - and He-like ions of $\mathrm{Si}, \mathrm{S}, \mathrm{Ar}, \mathrm{Ca}$, and $\mathrm{Fe}$, as well as an iron $\mathrm{K}$ complex from low-ionization Fe species (Kitamoto et al. 1994). The high absorption column density of Cyg X-3 cuts off the spectrum below $\sim 1.5 \mathrm{keV}$ and prevents the detection of other lines from e.g. $\mathrm{O}$ and $\mathrm{Fe} \mathrm{L}$. There were several unidentified line features in the SIS spectrum, in particular a feature at $\sim 3.5 \mathrm{keV}$. Liedahl and Paerels (1996) have reanalyzed the $A S C A$ data and identify the mysterious $3.5 \mathrm{keV}$ feature with the $\mathrm{S} X V I$ radiative recombination continuum (RRC). Liedahl and Paerels (1996) go on to fit the entire ASCA SIS spectrum with a model that includes for $\mathrm{Mg}, \mathrm{Si}, \mathrm{S}, \mathrm{Ar}, \mathrm{Ca}$, and $\mathrm{Fe}$ the first five members of the Lyman series of the hydrogenic ions, the He-like lines, and the $\mathrm{K}$-shell RRC (Figure 3). Blending of the He-like and H-like lines with the RRC 

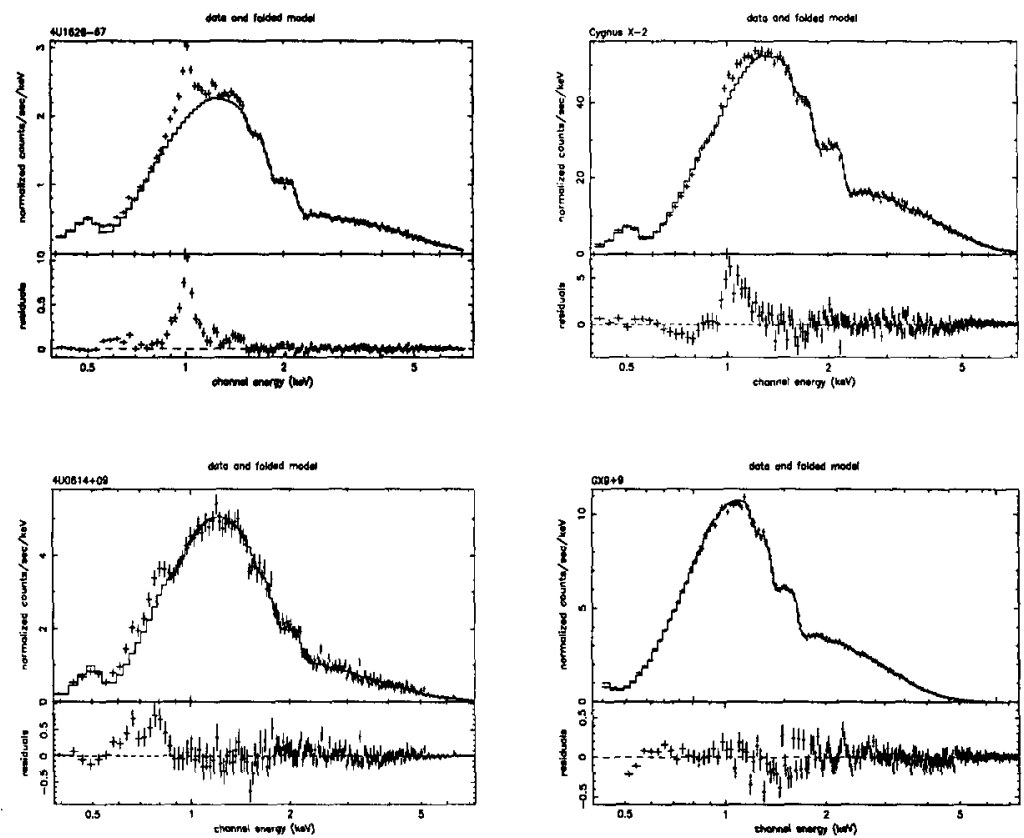

Figure 4. The $A S C A$ SIS spectrum of the $7 \mathrm{~s}$ pulsar $4 \mathrm{U} 1626-67 \mathrm{spec}-$ trum is dominated by a neon Ly- $\alpha$ line at $1 \mathrm{keV}$ (top left). The $Z$ source Cygnus X-2 shows a broad $1 \mathrm{keV}$ Fe L emission feature (top right). 4U0614+09 shows O Ly- $\alpha$ and Ly- $\beta$ emission (bottom left) and GX $9+9$ no line emission (bottom right).

features means that only the S XVI RRC is well resolved. But there is also good evidence for the Mg XII and Si XIV RRC in the spectrum. This is the first time that the RRC has been clearly identified in the spectrum of a cosmic X-ray source. The width of these lines constrains the electron temperature and gives $32-75 \mathrm{eV}$ for the $\mathrm{S}$ RRC line.

The two most likely models proposed for Cyg X-3 are scattering in a stellarwind from a Wolf-Rayet star (Pringle 1974; Davidson and Ostriker 1974), and an accretion disk corona occulted by the thickened edge of an accretion disk (White and Holt 1982). The recent ir identification of Cyg X-3 with a WolfRayet star (van Kerkwijk et al. 1992) favors the stellar-wind model. The rich line emission and RRC discovered by ASCA most probably originates in the $\mathrm{X}$-ray illuminated wind of the Wolf-Rayet star.

\section{Low Energy Line Emission from LMXRB}

The low energy line emission from LMXRB most likely originates in an ADC, and provides a useful tool to investigate the ADC structure. Figure 4 shows 
the spectra obtained using the $A S C A$ SIS from four LMXRB of different types. There are different low energy line emission patterns that seem to depend on the evolutionary history of the LMXRB (White, Kallman and Angelini 1996). The $7 \mathrm{~s}$ pulsar 4U1626-67 shows a strong narrow feature centered at $1 \mathrm{keV}$. Cygnus X-2, a luminous $Z$-source with a 9 day orbital period, shows a weaker and broader feature between 1 and $1.5 \mathrm{keV}$. The X-ray burst and and atoll source $4 \mathrm{U} 0614+09$ exhibits line features around $0.6-0.8 \mathrm{keV}$. GX9+9 has a relatively featureless spectrum, with no evidence for any low energy line emission.

The strongest features in the spectrum of 4U1626-67 are consistent with the $\mathrm{L} \alpha, \mathrm{L} \beta$, and the Lyman continuum of $\mathrm{Ne} X$, at $1.02,1.21$, and $1.36 \mathrm{keV}$ respectively (Angelini et al. 1995). These lines dominate over the expected iron $\mathrm{L}$ lines, which suggests an over-abundance of Neon by a factor of 10 . A Neon overabundance implies Helium burning has taken place in the companion star. This directly excludes the hypothesis of a hydrogen-depleted donor star in 4U1626-67 and instead suggests that the donor star in 4U1626-67 is a helium-burning star with a mass $\geq 0.5 M_{\odot}$ or a Neon rich white dwarf (Angelini et al. 1995). A broad line at $0.77 \mathrm{keV}$ detected in the Einstein SSS of 4 U0614+09 by Christian et al. (1993) is resolved by the ASCA SIS into two lines at $0.65 \mathrm{keV}$ and $0.80 \mathrm{keV}$ from $\mathrm{O}$ Ly $\alpha$ and $\beta$ emission. Similar oxygen emission is seen in $A S C A$ spectra of other related LMXRB systems e.g. 4U0918-54 and 4U1543-62 (Angelini et al. 1997). But in the case of Cyg X-2 there is a broad line emission feature centered on $1 \mathrm{keV}$ (Vrtilek et al. 1991 and Figure 4), consistent with a blend of Fe $\mathrm{L}$ emission lines. Model fitting to these spectra using the XSTAR code (Kallman and Krolik 1994) shows that in Cyg X-2 the oxygen abundance relative to iron is a factor of 10 below solar. Cyg X-2 has a 9 day orbital period with a companion star that has evolved from the main sequence. Kahn et al. (1984) suggest that convective mixing has dredged up processed material from the nuclear burning layers and then transferred them to the companion through the accretion flow. If $4 \mathrm{U} 0614+09$ is in a shorter period system, where the companion is less evolved, then the abundance pattern is expected to be different. No low energy line features are detected in GX9+9 with a 3 sigma upper limit of $<2-3 \mathrm{eV}$; a factor of 5-10 less than the features seen from 4U0614+09 and Cyg $\mathrm{X}-2$. This might reflect different conditions in the emission region in $\mathrm{GX} 9+9$, but another possible explanation is that the overall abundances of both iron and oxygen are reduced because this is an old population II object. All the spectra shown in Figure 5 do not show strong RRC features. Angelini et al. (1995) discuss a possible Ne X RRC feature in the spectrum of 4U1626-67. But this feature is only marginally detected, confused with a $\mathrm{Mg}$ line and in fact the line emission is consistent with a fit to a collisional plasma. This is also the case for the spectra from 4 U0614+09 and Cyg X-2. Angelini et al. (1995) suggest that resonant scattering of the photoionized emission washes out the recombination edges.

\section{5. $A S C A$ Observations of the ADC sources}

The 1-7 keV continuum of the ADC sources is much harder than the typical continuum of LMXRB observed face on. The photon index of 0.5 from 4U1822-37 compares to a more typical value of $\sim 1.5-2.5$ in more typical burst sources 


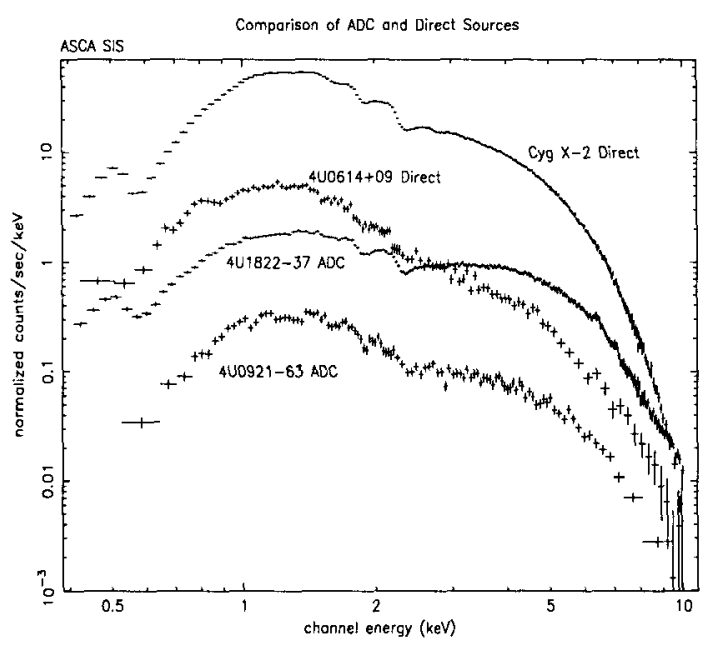

Figure 5. A comparison of LMXRB spectra viewed at different inclination angles. The two high inclination ADC sources 4U1822-37 and 4U0921-63 (Mason et al. 1987) have orbital periods of $5.57 \mathrm{hr}$ and 9 days respectively. The sources $4 \mathrm{U} 0614+09$ and Cyg X-2 are the low inclination counterparts to these systems. Note that in both ADC sources the spectrum is harder than the face on systems.

(White, Stella and Parmar 1988). In Figure 5 the spectrum of 4 U1822-37 is compared to that of a face-on systems $4 \mathrm{U} 0614+09$. They are quite clearly different, with the spectrum of the ADC source much harder. A similar trend is seen for another ADC source $4 \mathrm{U} 0921-63$. This is a 9 day binary and may be a high inclination version of Cyg X-2. Again the spectrum of the ADC source is harder than its face on counterpart, with a relatively featureless spectrum and a high energy cutoff around the iron K edge. The fact that in the ADC systems there is no strong counterpart to the low energy line emission seen from LMXRB systems viewed face on (Figure 4), suggests the line production region is very close to the X-ray source, in the central regions that are not seen in the high inclination systems.

The spectrum scattered by the ADC should to first order reflect that of the central source, unless the optical depths are large. In both cases shown in Figure 5, the ADC spectra are quite clearly much harder than their face on counterparts. The ASCA SIS and GIS spectra of 4U1822-37 are shown in Figure 6 , with a fit to a simple power law with an index of $\sim 0.52$. The residuals show three features: 1) a break in the spectrum at $\sim 7 \mathrm{keV}$; 2) a minimum $\sim$ $1.5 \mathrm{keV}$; and 3 ) no strong emission line features. Figure 5 also shows a more detailed view of the iron $\mathrm{K}$ line region. Two line features are evident at 6.4 $\mathrm{keV}$ and $7.1 \mathrm{keV}$ (with a possible third weaker line feature at $6.6 \mathrm{keV}$ ). The 6.4 and $7.1 \mathrm{keV}$ line energies match those of the iron $\mathrm{K} \alpha$ and $\mathrm{K} \beta$ lines. The equivalent width of the $6.4 \mathrm{keV}$ line is $\sim 40 \mathrm{eV}$. However, the $\mathrm{K} \beta / \mathrm{K} \alpha$ ratio of $\sim 40 \%$ does not match the theoretical value of $13 \%$. An alternate explanation 

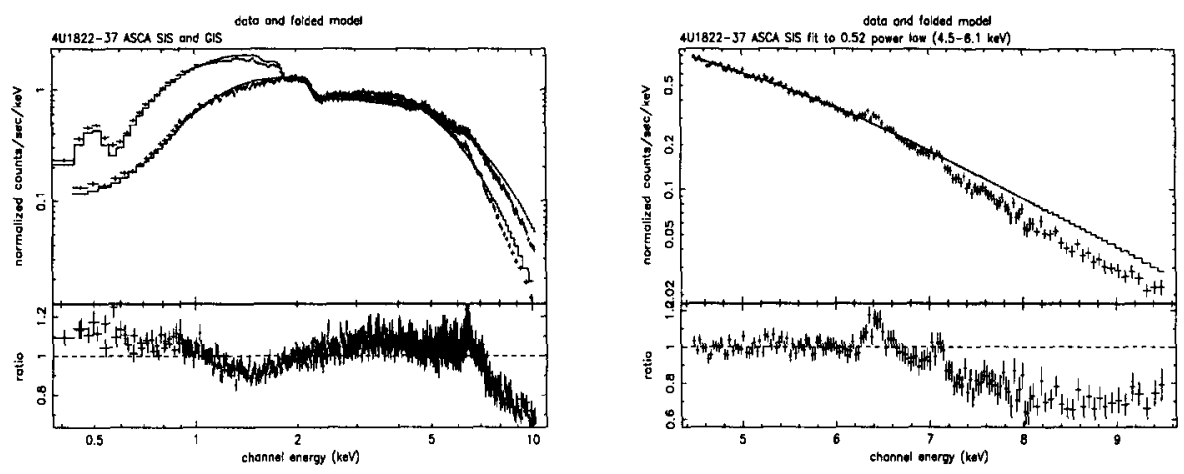

Figure 6. The ASCA SIS and GIS spectra of 4 U1822-37 when fit to a simple power law model with a photon index of 0.52 . The upper part of the left panel shows the entire spectrum and model fit. The upper part of the right panel shows an expaned view of the iron $K$ region. The residuals are shown in the lower two panels. There is an abrupt decline in the spectrum around the iron $\mathrm{K}$ edge at $7.1 \mathrm{keV}$. A small iron $\mathrm{K} \alpha$ line is evident at $6.4 \mathrm{keV}$ and a feature around $6.8 \mathrm{keV}$.

is that instead of an emission line at $7.1 \mathrm{keV}$, there is a resonant absorption line at $\sim 6.9 \mathrm{keV}$. The continuum rapidly decreases above $7.1 \mathrm{keV}$, i.e. around the iron $\mathrm{K}$ absorption edge. This decline is too broad for a single edge, and instead the sum of edges from various ionization stages of iron are required to explain the observed spectrum. Constraining the continuum above $10 \mathrm{keV}$ is crucial to constrain the iron $\mathrm{K}$ absorption feature evident in Figure 6 . We have tried to use archival Ginga and EXOSAT data to do this, but do not obtain a satisfactory solution using these non-simultaneous data.

The edge feature around the iron $K$ region suggests that reflection from the accretion disk may be important (e.g. Ebisawa et al. 1994). However, while disk reflection models can provide a satisfactory fit over a limited band $(5-10$ $\mathrm{keV}$ ), they fail when the entire $1-10 \mathrm{keV}$ spectrum is considered because they predict a sharp fall off at low energies, and/or strong low energy line emission. Disk reflection also predicts a huge iron $K \alpha$ line that is not observed in the ADC spectra. A more likely explanation is partial covering, which can produce a hard spectrum. This model provides a reasonable fit to the $4 \mathrm{U} 1822-37$ spectrum, but does require a broad iron line. This broadening may be due to Compton scattering or rotation in the inner disk (see e.g. White and Holt 1982; Kallman and White 1989). Currently the spectra of the ADC sources remains puzzling and further theoretical modelling is required, together with observations that extend above $10 \mathrm{keV}$ to better constrain the underlying continuum.

Acknowledgments. I thank my collaborators Tim Kallman and Lorella Angelini for discussions and for providing data in advance of publication. 


\section{References}

Angelini, L., et al. 1995, ApJ, 449, L41.

Angelini, L., et al. 1997, in preparation.

Armitage, P.J. and Livio, M., 1996, ApJ, in press.

Christian, D., White, N.E. and Swank, J.H. 1993, ApJ, 422791

Cominsky, L., and Wood, K. 1984, ApJ, 283, 765

Davidson, K. and Ostriker, J.P. 1974, ApJ, 189, 331

Ebisawa, K. et al. 1994, PASJ, 46, 375

Frank, J., King, A. R., and Lasota, J-P, 1987, A\&A, 178, 137

Hellier, C. and Mason, K.O. 1989, MNRAS, 239, 715

Hirano, T., et al. 1987, PASJ, 39, 619

Kahn, S. M., Seward, F. D., and Chlebowski, T. 1984, ApJ, 283, 286

Kallman, T. R., and Krolik, J. H. 1994, NASA/GSFC/LHEA preprint

Kallman, T. and White, N.E. 1989, ApJ, 341, 955

Kitamoto, S. et al. 1994, PASJ, 46, L105

Ko, Y., and Kallman, T.R., 1994 ApJ, 431, 273

Liedahl, D., Kahn, S.M., Goldstein, W., and Osterheld, A., 1992 ApJ, 391, 306

Liedahl, D., and Paerels, F., 1996, ApJ, 468, L33

Lubow, S. H., and Shu, F. H. 1975, ApJ, 198383

Mason, K.O., and Córdova, F.A. 1982, ApJ, 262, 253.

Mason, K. O., Branduardi-Raymont, G., Córdova, F. A., and Corbet, R. H. D. 1987, MNRAS, 226, 423

Milgrom, M. 1978, A\&A, 208, 191

Parmar, A.N., White, N.E., Giommi, P., and Gottwald, M. 1986, ApJ, 308, 199

Pringle, J.E. 1974, Nature, 247, 21

Shakura, N.I. and Sunyaev, R.A. 1973, A\&A, 24, 337

Suzuki, K., et al. 1984, PASJ, 36, 761

van Kerkwijk, M. H., et al. 1992, Nature, 355, 703

Vrtilek, S. D., Helfand, D. J., Halpern, J. P., Kahn, S. M., and Seward, F. D. 1986, ApJ, 308, 644

Vrtilek, S.D., et al. 1991, ApJSupp, 76, 1127.

White, N.E., et al. 1981, ApJ, 247, 994

White, N.E., and Holt, S.S. 1982, ApJ, 257, 318

White, N.E., Kallman, T.R. and Angelini, L., 1996, in X-ray Imaging and Spectroscopy of Cosmic Hot Plasmas, ed. F. Makino (ISAS), in press

White, N.E., Nagase, F., and Parmar, A.N. 1995, in $X$-ray Binaries, eds. W.H.G. Lewin, J. van Paradijs, \& E.P.J. van den Heuvel (Cambridge: CUP), p1

White. N.E., Stella, L., and Parmar, A.N., 1988, ApJ, 324, 363

White, N.E., et al. 1986, MNRAS, 218, 129 